\title{
Equipment and procedural setup for interventional pulmonology procedures in the intensive care unit
}

\author{
Amrik S. Ray ${ }^{1}$, Van K. Holden ${ }^{2}$, Ashutosh Sachdeva ${ }^{2}$, Faria Nasim ${ }^{2}$ \\ ${ }^{1}$ Chicago Chest Center, Suburban Lung Associates, Elk Grove Village, IL, USA; ${ }^{2}$ Division of Pulmonary \& Critical Care Medicine, University of \\ Maryland School of Medicine, Baltimore, MD, USA \\ Contributions: (I) Conception and design: All authors; (II) Administrative support: All authors; (III) Provision of study materials or patients: All \\ authors; (IV) Collection and assembly of data: All authors; (V) Data analysis and interpretation: All authors; (VI) Manuscript writing: All authors; (VII) \\ Final approval of manuscript: All authors. \\ Correspondence to: Faria Nasim, MD. Division of Pulmonary \& Critical Care Medicine, University of Maryland School of Medicine, Baltimore, MD, \\ USA. Email: FNasim@som.umaryland.edu.
}

\begin{abstract}
Procedural setup is an important aspect of any procedure. Interventional pulmonologists provide a procedural practice and have additional expertise in performing high-risk procedures needed in the critically ill patients in intensive care. Taking the time to plan the procedure setup in advance and having all necessary equipment readily available at the patient's bedside is imperative for procedural services. This is especially essential to ensure patient safety, minimize risk of complications, and improve success for specialized procedures performed by interventional pulmonary in the intensive care unit. In this review we describe the equipment and procedural setup ideal for both pleural and airway procedures. These include flexible diagnostic and therapeutic bronchoscopy, ultrasound guided thoracentesis, chest tube insertion, difficult airway management, and bedside percutaneous dilatation tracheostomy. We provide a guide checklist for these procedures emphasizing the practical aspects of each procedure from selecting the appropriate size endotracheal tube to operator positioning to ensure efficiency and best access. The components of procedural setup are discussed in relation to patient factors that include patient positioning and anesthesia, personnel in the procedure team and the equipment itself. We further briefly describe the additional equipment needed for specialized techniques in therapeutic bronchoscopy used by interventional pulmonologists.
\end{abstract}

Keywords: Procedural setup; interventional pulmonary; bronchoscopy equipment; bedside intensive care unit procedures (bedside ICU procedures)

Submitted Dec 30, 2020. Accepted for publication Mar 02, 2021.

doi: $10.21037 /$ jtd-20-3595

View this article at: http://dx.doi.org/10.21037/jtd-20-3595

\section{Introduction}

Interventional pulmonology (IP) is frequently consulted to perform procedures in the intensive care unit (ICU) setting. At many centers, these procedures are performed by intensivists or physicians from other sub-specialties. An IP team designed to streamline procedural practice in an academic setting serves to improve efficiency, reduce procedural time and decrease procedural complications (1). Depending on the type of procedure required, different equipment setup is necessary. Frequently performed procedures include diagnostic and therapeutic bronchoscopy, thoracentesis, chest tube insertion, airway management, and percutaneous dilatation tracheostomy. Most patients admitted to the ICU have significant co-morbidities. As such, they are inherently at risk for procedure related complications. Having a dedicated procedural team and a systematic approach to procedural equipment and setup minimizes risk and ensures successful outcome. 


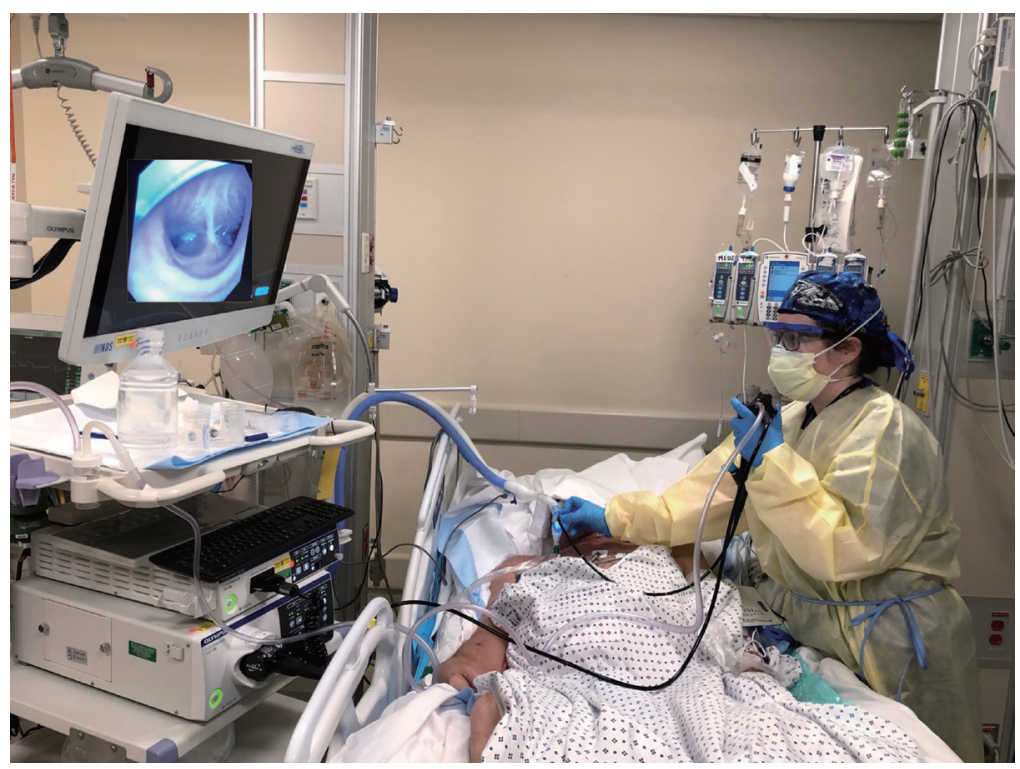

Figure 1 Bedside bronchoscopy setup in an intensive care unit with the operator at the side of the bed. Notice the inverted carina in the bronchoscopic image with the left mainstem visualized on the right side of the monitor.

\section{Flexible bronchoscopy (FB) procedure}

\section{Bronchoscopy procedural set-up}

FB is frequently performed in an ICU setting. The first and most important step is to ensure that all needed equipment and supplies are readily available. The bronchoscopy tower or disposable bronchoscope screen should be setup at the patient's bedside in the ICU. The patient is placed in a supine position with the ventilator in a controlled mode. Anecdotally, patients' fraction of inspired oxygen $\left(\mathrm{FiO}_{2}\right)$ is increased to $100 \%$ for the duration of the procedure. FB is typically done at the head of the bed, though it is often also done from the side of the bed especially in intubated patients in ICU. When the operator is at the head end of the bed, the bronchoscopic image visualized on the monitor shows the left mainstem on the left side of the monitor, and the anterior tracheal wall is on the upper part of the screen. This contrasts with the bronchoscopic image when the operator stands at the side of the bed and the bronchoscopic image on the monitor is inverted. With the operator at the side of the bed, the patient's left mainstem is seen on the right side of the monitor and the posterior tracheal wall is now on the upper part of the screen (Figure 1). With the image inversion, the bronchoscopist adjusts for anatomy when performing any therapeutic interventions. In order to create space for the bronchoscopist at the head end of the bed, the patient's bed, ventilator and intravenous line setups need to be moved. The patient's vital signs monitor is also adjusted in line with the proceduralist's visual field to allow real time patient vital signs monitoring. At the beginning of every procedure, a bite block should be placed to prevent damage to the bronchoscope irrespective of the patient's level of sedation or use of neuromuscular blockade.

Typically, two suctions are setup, one for the bronchoscope and a second for oral suctioning. After setting up all equipment including the swivel adaptor and bite block, a time out is performed. Then sedation and the procedure can begin. In intubated patients, the trachea, left and right mainstem bronchus are anesthetized by topical lidocaine to decrease coughing and subsequent decruitment of the lungs during the procedure. A FB procedure is further classified as diagnostic, therapeutic or both.

\section{Diagnostic bronchoscopy}

Diagnostic bronchoscopy is often performed with standard bronchoscopes [outer diameter (O.D.) 5.0- and 2.0-mm working channel] and as such can be done through smaller endotracheal (ET) tubes. When done via an ET tube or laryngeal mask airway (LMA), the bronchoscope must pass through a swivel adaptor to enter the airway to maintain a seal for ventilation (Figure 2).

Flexible bronchoscopes range in O.D. from 3.0 to $6.0 \mathrm{~mm}$. When inserted into an ET tube, a clearance 


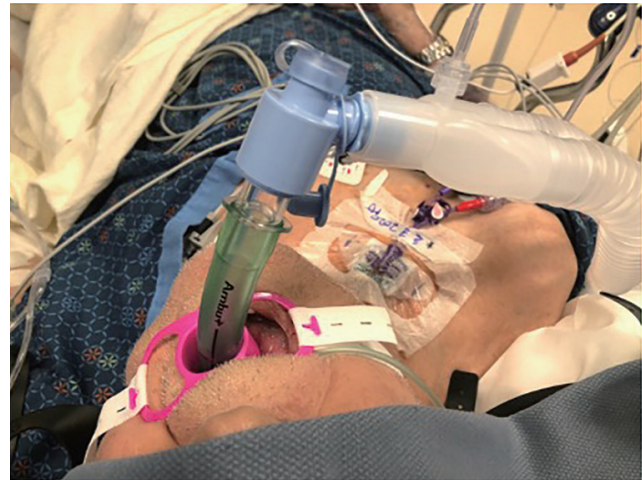

Figure 2 An example of a bronchoscope set up with a ventilator connected to an LMA via a swivel adaptor. Note the bite block present around the LMA to prevent scope damage. LMA, laryngeal mask airway.

of $>2 \mathrm{~mm}$ between the O.D. of the bronchoscope and inner diameter (I.D.) of the ET tube is recommended. This prevents high airway pressures, ensures adequate ventilation, and avoids scope damage $(2,3)$. If a larger diameter therapeutic flexible bronchoscope is required for a procedure (e.g., removal of blood clots), and the patient has a smaller than adequate ET tube, then upsizing the ET tube will be necessary.

For diagnostic procedures, the most frequent equipment needed is setup for a bronchoalveolar lavage. These are usually 20 or $60 \mathrm{cc}$ slip tip syringes that insert into the biopsy port on a bronchoscope. If a slip tip syringe is not available, a Luer lock syringe can be used with an adaptor. Alternatively, a Luer lock syringe can be modified by simply cutting off the threaded portion which results in a slip tip syringe. In addition, adequate syringes for lidocaine administration will be necessary. The lidocaine administration is usually via $10 \mathrm{cc}$ slip tip syringes. Syringes with lidocaine should include air at the top to ensure the contents of the syringe are flushed completely through the working channel.

In recent years, single use disposable flexible bronchoscopes have been introduced. Disposable scopes differ from traditional flexible bronchoscopes in that they have an integrated video processing unit/light source and do not require an external source. Disposable scopes are being used for airway inspection, bronchoalveolar lavage and secretion management. Disposable scopes are used for biopsies in limited circumstances. Disposable scopes are cost effective in lower volume hospitals where $<200 \mathrm{FBs}$ are performed every year $(4,5)$.
In some circumstances, diagnostic biopsies of a target lesion are needed. A target lesion would have been noted on a preprocedural computerized tomography chest or incidentally found during the airway exam. Diagnostic biopsies can be performed with direct visualization via a bronchoscope with forceps. Alternatively, if the lesion is peribronchial or mediastinal then via utilization of a linear endobronchial ultrasound (EBUS) equipped bronchoscope. If needed, the bronchoscopist will employ biopsy tools such as a transbronchial needle and forceps. Of note, the standard linear EBUS bronchoscopes diameter is about 6.5-7.0 $\mathrm{mm}$. These EBUS scopes require size 8.5-9.0 ET tubes to ensure adequate ventilation. Forceps, brushes, and transbronchial needle aspiration needles can be used both endobronchially and transbronchially. For any procedure with parenchymal biopsies, it is essential to have a smallbore chest tube kit readily available at the bedside, should a pneumothorax ensue.

\section{Therapeutic bronchoscopy}

In addition to the equipment described above, patients typically require a larger flexible bronchoscope with a 5.8-6.4 mm diameter typically referred to as a therapeutic scope. These patients need an even larger ET tube to accommodate the therapeutic FB and simultaneously ensure adequate tidal volumes with mechanical ventilation.

One of the most common indications for FB in an ICU setting is thick secretions leading to mucus plugging of the airways with subsequent lobar collapse, hypoxemia and difficulty managing the mechanical ventilator. For dense mucus plugging, the most important tool is excellent wall suction setup. Saline and topical medications such as $\mathrm{N}$-acetylcysteine have been reported to help clear thick secretions $(6,7)$. Cryoprobes are frequently used to facilitate extraction of particularly tenacious mucus plugs (8).

In the patient with overt or suspected hemoptysis, additional equipment is setup. For actively bleeding endobronchial lesions, a thermal ablation device is used to locally control the bleeding vessel. Most commonly argon plasma coagulation and electrocautery are used. To prevent airway fires, $\mathrm{FiO}_{2}$ should be decreased to less than $40 \%$ when using a thermal ablative device. Cryoprobes are an excellent tool to extract larger organized blood clots, often en bloc (9). If active bleeding is noted on the initial airway exam with poor visualization of the airways and a location for the bleeding cannot be identified to intervene, a balloon occlusion device can be used to tamponade bleeding 


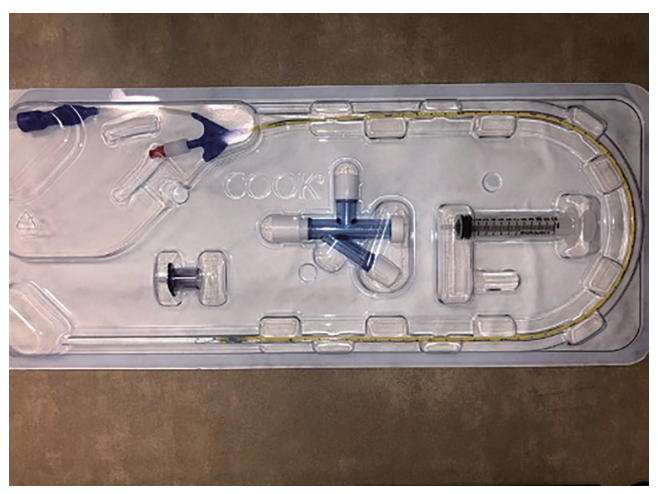

Figure 3 Arndt Bronchial Blocker. The appropriate swivel adaptor is in the center with the blocker itself wrapped around the edge of the packaging. The device in the center left of the screen is an adaptor for jet ventilation.

Table 1 Devices for different therapeutic bronchoscopy indications

\begin{tabular}{lcccc}
\hline Devices & $\begin{array}{c}\text { Mucus } \\
\text { plugging }\end{array}$ & Hemoptysis & $\begin{array}{c}\text { Foreign } \\
\text { body }\end{array}$ & $\mathrm{CAO}$ \\
\hline Cryo probe & $\mathrm{X}$ & $\mathrm{X}$ & $\mathrm{X}$ & $\mathrm{X}$ \\
Thermal devices & & $\mathrm{X}$ & & $\mathrm{X}$ \\
Balloon occlusion & $\mathrm{X}$ & & $\mathrm{X}$ \\
CRE/Balloon dilation & & & $\mathrm{X}$ \\
Extracting devices & & $\mathrm{X}$ & $\mathrm{X}$ \\
Stenting equipment & & & $\mathrm{X}$ \\
\hline
\end{tabular}

CAO, central airway obstruction.

airways. In such cases of massive and difficult to control bleeding, an Arndt Endobronchial Blocker is deployed. Arndt Bronchial Blocker comes in 3 sizes namely 5F, 7F, and 9F. These have a special swivel adaptor with a separate port for entry of the blocker itself. This adaptor and Arndt Endobronchial Blocker kit are shown in Figure 3. For the largest size, a patient will need a size 9 ET tube along with a small bronchoscope such as a diagnostic, pediatric, or thin bronchoscope to allow room for passage of the blocker. Topical medications used to control airway bleeding include epinephrine, iced saline (10), tranexamic acid (11), and thrombin (12).

For a foreign body extraction, various extracting devices along with a large ET tube or rigid bronchoscope may be needed in rare circumstances. The various devices include baskets of various sizes, balloons (to pass through or behind lesions), forceps-especially large rat tooth forceps, and cryoprobes (if the foreign body has a high water content) (13).

Lastly therapeutic bronchoscopy may be needed to address central airway obstruction. All the aforementioned equipment can be used for the management of airway obstruction. Most notably, are thermal devices, cryoprobes, and airway balloons $(14,15)$. In addition to occlusion balloons, controlled radial expansion (CRE) balloons are often used to dilate the area of obstruction. In some circumstances, stents are deployed. Depending on the size of the stent, FB alone can sometimes suffice for placement of self-expanding metallic stents. Rigid bronchoscopy (RB) is used in cases of severe malignant airway obstruction where FB alone will be unsuccessful in recanalization of the airway. In these cases, the ET tube is removed, and a rigid bronchoscope is placed expeditiously. RB setup consists of a beveled metal tube, an external light source, video processing equipment, a telescopic camera, rigid therapeutic tools and a jet ventilator. The rigid scope itself takes the place of a standard ET tube and a standard jet ventilator is attached to it. RB is used sparingly in the mechanically ventilated patient in the ICU by skilled interventional pulmonologists or thoracic surgeons. A summary of the various types of devices used for therapeutic bronchoscopy and their indications are listed in Table 1.

\section{Pleural procedures}

Commonly performed procedures in the ICU by interventional pulmonologists are ultrasound guided thoracenteses, chest tube thoracostomy, and rarely tunneled pleural catheters. The most frequently used ultrasound probes in pleural procedures are a high frequency linear probe (primarily to assess for pneumothoraces) and a phased array probe (to assess pleural fluid) (Figure 4).

\section{Pleural procedural set-up}

The equipment is typically setup at the side of the procedure being performed. The US is setup either on the same side or on the other side of the patient and across the operator based on the operator's preference. The goal is to give the operator in line vision of the US image and easy access to the equipment (Figure 5). Similarly, positioning of the patient is equally important. In intubated patients, the lateral decubitus position with the head of the bed elevated 


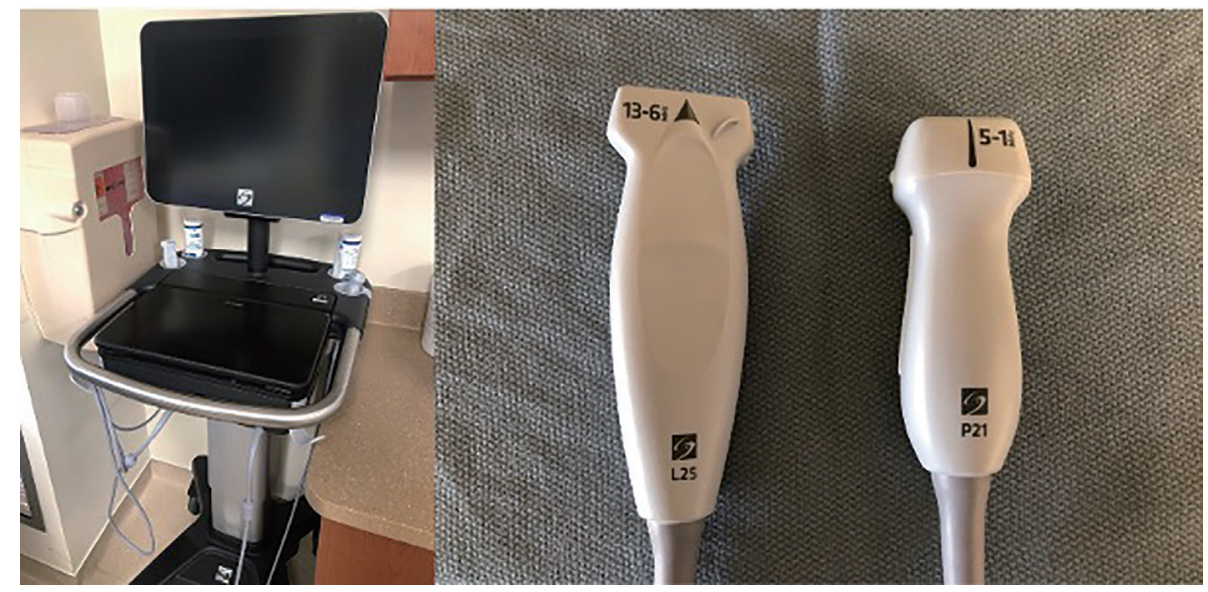

Figure 4 Point of Care SonoSite Ultrasound on top. Two probes visible below, with a high frequency linear transducer on the left and a small footprint phased array transducer on the right.

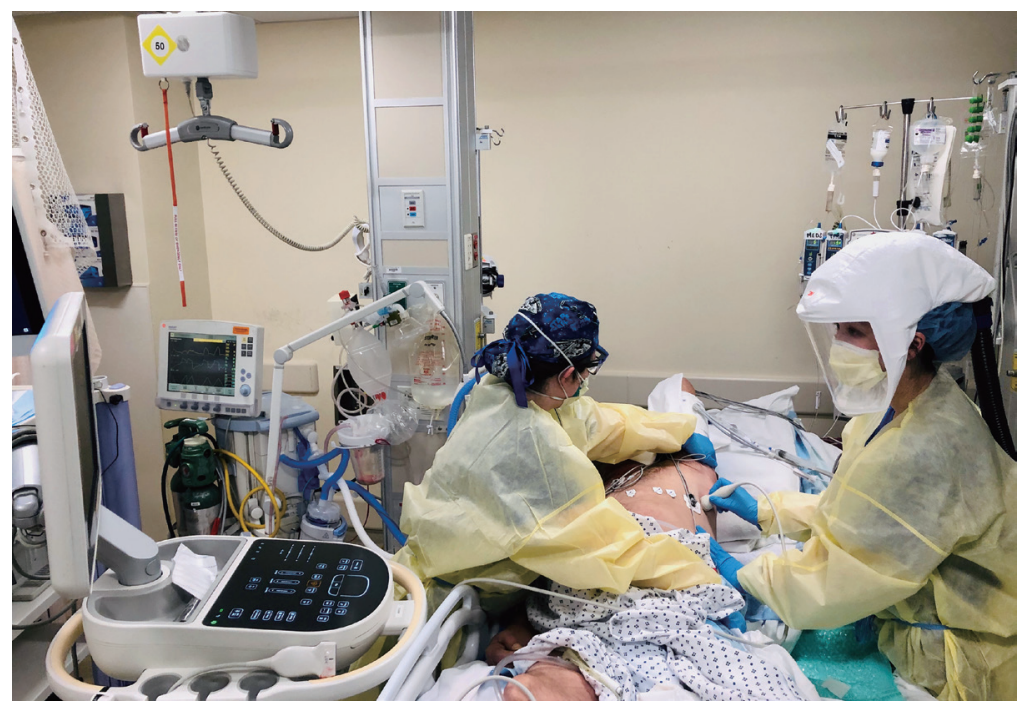

Figure 5 Bedside setup for pleural ultrasound prior to thoracentesis in an intensive care unit.

is a commonly used position. This allows for access to identify an ultrasonic pocket of pleural effusion and space posteriorly to perform a safe thoracentesis. In some cases, the patient can sit supported at the edge of the bed. With ventilators this position may be more difficult to arrange and requires additional support staff. Percutaneous chest tubes and tunneled pleural catheters are sometimes done with the patient seated, though more often they are done with the patient in a supine or lateral decubitus position with the head slightly elevated. Surgical chest tubes are typically done with the patient in supine or lateral decubitus position. It is preferable to have the patient lie on a towel roll to help open the rib spaces for the procedure. After positioning, the pleural space is examined with US and the planned site of entry is marked. A time out is performed and the procedure can be started.

\section{Thoracentesis}

Several thoracentesis trays are commercially available for use and are stocked in inpatient and outpatient settings. These trays are single use and typically include all the key 
equipment needed for the procedure. The prepackaged disposable tray includes chlorhexidine, a fenestrated drape, needles, syringes, an access catheter, sterile gauze, blade, suction tubing, a bag for collection of fluid, 3 specimen vials and a dressing. A filter needle is provided to draw lidocaine. In some kits a vial of lidocaine is included. Most kits have an 8-Fr access catheter typically loaded over a $18 \mathrm{G}$ introducer needle. The dressing is typically a larger size occlusive bandage. For a diagnostic thoracentesis where $<50 \mathrm{cc}$ of fluid is to be aspirated, it is possible to do this with only a $21 \mathrm{G} / 22 \mathrm{G}$ needle and a syringe after cleaning under local anesthesia. For the operator, gowns, gloves and facemask are arranged separately. If the drainage of pleural fluid is expected to exceed $2 \mathrm{~L}$, additional drainage containers will have to be setup before starting the procedure. Fluid can be safely aspirated using gravity drainage, manual aspiration or active suction. The prepackaged trays include equipment to setup gravity drainage with bag and 3 way stop cock for manual aspiration. Active suction can be performed using vacuum bottles or low wall suction. To establish drainage under low wall suction, tubing and canister setup like nasogastric tube aspiration is setup and constant pressure of 0 to $-80 \mathrm{~mm} \mathrm{Hg}$ is used. Sterile gloves and mask are the minimum protective gear for the operator.

\section{Chest tube}

Chest tubes can be divided into percutaneous small-bore $(<14 \mathrm{Fr})$ chest tube and larger bore surgical chest tube. Larger bore surgical chest tubes have largely fallen out of favor in the medical ICU as all indications for a chest tube can be addressed with the use of a small-bore chest tube with no difference in patient outcomes. The two most common indications for chest tube placement in an ICU include pneumothorax and empyema. Percutaneous small-bore chest tubes are flexible and available in a variety of prepackaged kits. These have similar contents to the thoracentesis kits with the addition of the percutaneous catheter and a dilator. For any chest tube, a disposable underwater-seal drainage chamber will need to be separately setup. Pleur-evac ${ }^{\circledR}$ is commonly used in the United States and is designed for drainage of the pleural cavity. This is connected to wall suction at $-80 \mathrm{mmHg}$ per the manufacturing recommendations. In some circumstances, additional draping materials are needed as well. Sterile gloves and mask are the minimum protective gear for the operator.
Surgical chest tubes are larger bore and straight. There are no commercially available kits for large bore chest tubes. The supplies for surgical chest tubes are often stocked in a chest tube cart readily available in most ICUs. For an open surgical chest tube, the practitioner will need to establish a preprocedural setup. These should include disinfectant such as providone-iodine or chlorhexidine, local anesthetic commonly lidocaine, drapes, scalpel (\#10/\#11 blade), skin retractors, chest tube, drainage system, Christmas tree adaptor, 2 or 3 way stop cock, suture, needle driver, forceps, gauze, and dressing supplies. Full sterile gown, cap, gloves and mask are worn by the operator and full sterile techniques are used unless an emergency dictates otherwise.

\section{Tunneled pleural catheter}

If a recurrent malignant pleural effusion is encountered in the ICU, an indwelling tunneled pleural catheter can be placed. These are typically done via percutaneous insertion using the a catheter kit. The preprocedural setup like chest tube thoracostomy. Additionally, two points of entry on the skin are marked before time out. Drainage System bottles and training videos are provided to the patient's family or nursing staff for future drainage of pleural fluid in the ICU.

\section{Difficult airway management}

FB is a valuable tool in the setting of high-risk intubation. This is particularly the case with patients with limited neck movement, small mouth opening, and potential upper airway stenosis. In these settings, direct laryngoscopy may be challenging, and FB is an appropriate option to help with both evaluation of the upper airway/trachea and to facilitate intubation. Too small a bronchoscope may prove to be too flexible to intubate over and too large of one may be difficult to maneuver in the patients' oropharynx (16). In addition to this, a bite block is important to have in addition to an appropriately sized ET tube (17). Direct semirigid and rigid fiberscopes (e.g., Bonfils intubation fiberscope) are also used as an alternative to $\mathrm{FB}$, but are less readily available in many institutions (18).

\section{Fiberoptic intubation procedural set-up}

The bronchoscopy tower should be setup at the bedside. However, in this circumstance, having the practitioner at the head of the bed may be especially important. It is also 


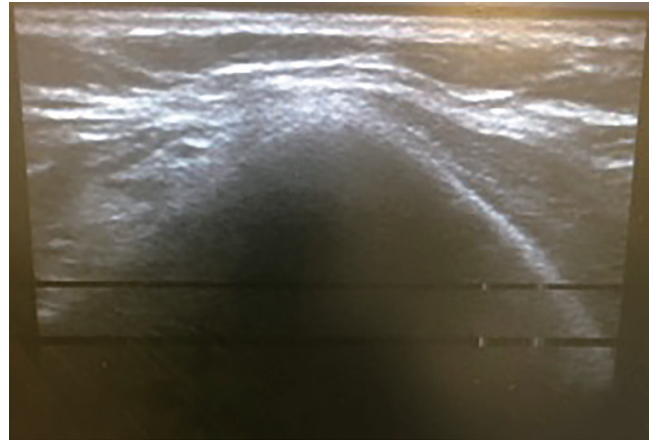

Figure 6 Tracheal ultrasound showing the tracheal air show in the middle. Note the absence of any large vessels or significant thyroid tissue.

essential to have two suctions (one for the bronchoscope and one for oral suctioning). After all equipment is available and ready, the patient is preoxygenated per protocol and a bite block is placed. The bronchoscope should be preloaded with an ET tube on the outside of it. It is important to well lubricate the scope and the ET tube. If the patient's mouth opening is too small to place a bite block, one can create a "lollipop" out of multiple pieces of gauze and use that as an ad hoc bite block. In rare circumstances a 2-operator technique is employed. In these circumstances the intensivist (operator \#1) inserts the laryngoscope to aid in visualization of the airway by mobilizing oropharyngeal soft tissue. The interventional pulmonologist (operator \#2) can then insert the bronchoscope loaded with the ET tube.

\section{Percutaneous dilatational tracheostomy (PDT)}

PDT is a commonly performed procedure in most ICUs. Upwards of $15 \%$ of PDT in ICU are performed by nonsurgical critical care physicians. An IP team designed to streamline PDT practice in an academic setting serves to improve efficiency, reduce procedural time and decrease procedural complications (1). Bronchoscopy is commonly performed at the time of PDT as adjunctive endoscopic visual guidance during the procedure. Most commonly, this is done flexibly, but some practitioners do use rigid bronchoscopes in rare circumstances-most commonly when airway obstruction is present or with very significant expiratory central airway collapse (19). FB is used for direct visualization of the airway and ET tube during tracheostomy needle placement, dilation and final tracheostomy tube placement. Bronchoscopy when used to localize the location for needle entry within the trachea prevents accidental cannulation into mediastinal structures. However, there is currently some uncertainty regarding the exact role FB during PDT as it adds complexity to the procedure, necessitates a second bronchoscopist, adds cost, and is associated with some negative physiologic consequences such as de-recruitment with subsequent hypoxemia (20). Recent studies question the need for routine bronchoscopy in low-risk patients, especially with the ubiquity and rise of use of ultrasound at time of bronchoscopy (20-23). These studies have reported that complication rates and success of PDT is essentially identical under ultrasound guidance alone as compared to when FB is used. However, these studies are in general, small number of patients. High risk and obese patients are more likely to benefit from bronchoscopic visualization.

Bedside ultrasound has emerged as a new addition to assist PDT. Neck ultrasound is informative with regards to preoperative identification of vascular anatomy of the neck, localization of tracheal rings and thyroid isthmus (24). When ultrasound is utilized, a representative tracheal ultrasound image should be obtained (Figure 6). Typically, a high frequency linear ultrasound probe is used to visualize the trachea. Even when used as only an adjunct, pre-procedural ultrasound changes the entry location $25-50 \%$ of the time $(25,26)$. FB and US can be used continuously or intermittently with no reported difference in outcomes (27). Figure 7 shows the setup and two operator positions for PDT in an ICU. To expedite these cases, the intensivist performs bronchoscopy while the interventional pulmonologist places the tracheostomy.

\section{Tracheostomy procedural set-up}

Mechanical ventilation is largely maintained by an ET tube but a LMA is an acceptable alternative. Patients are routinely sedated and paralyzed at the beginning of the procedure. The patient's sedation is increased if the patient is not adequately comfortable on the ventilator. The $\mathrm{FiO}_{2}$ on the ventilator is increased to $100 \%$. The next important step is to position the patient. This requires the patient to be supine and to have a large neck roll under the lower cervical spine/shoulders to establish passive neck hyperextension. This will minimize the soft tissue that the operator will have to go through to place the tracheostomy. After equipment is setup and the patient is positioned a time out is performed. 


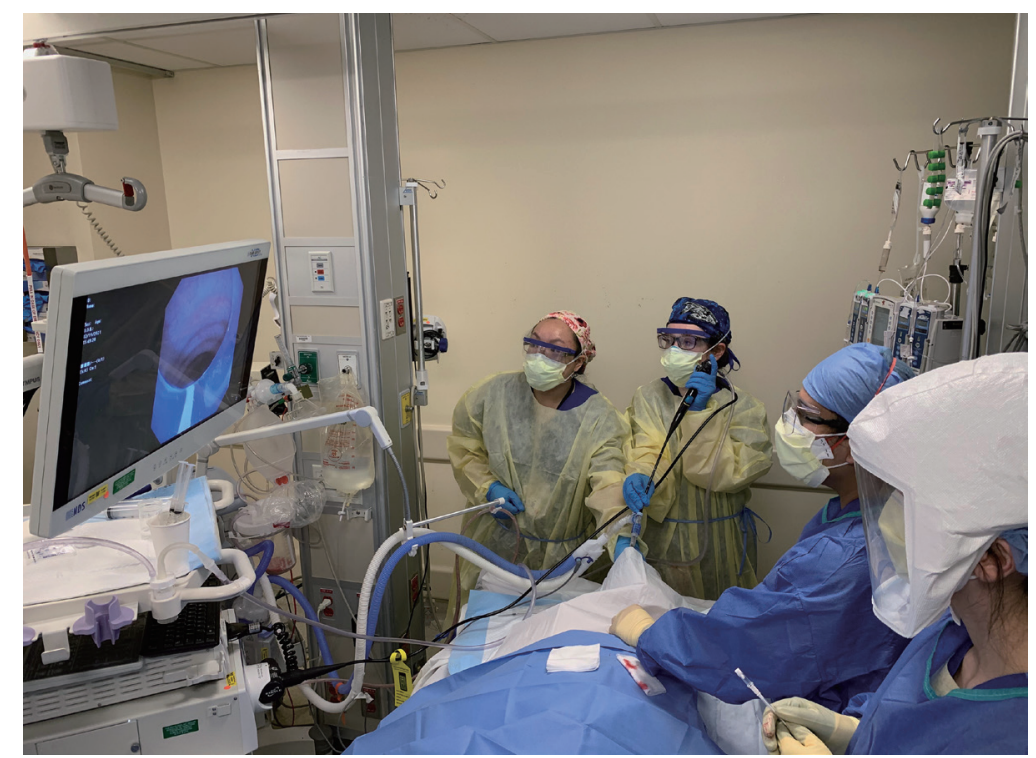

Figure 7 Percutaneous dilatational tracheostomy being performed with bronchoscopic guidance in an intensive care unit.

PDT can be performed in an ICU, at the bedside, with dilatation of the trachea over a guidewire followed by insertion of a tracheal cannula using Seldinger technique. PDT kits are readily available in the ICUs. Ciaglia Blue Rhino (Cook Critical Care, Bloomington, IN, USA) kit was introduced in the market in 1999 and the Ciaglia technique is the most commonly used technique in North America and worldwide (28). Dilation occurs in a two-step manner, first with a small punch dilator to create a gap in the trachea then via a larger single-beveled curved hydrophilic dilator; both are in the kit. Another commercially available kit is the PercuTwist (Rüsch, Teleflex, Athlone, Ireland). PercuTwist is a single-step screw-type dilator over a guidewire. In both these two kits, the use of a single dilator is associated with reduced tidal volume loss during the procedure (29). Portex Griggs percutaneous dilatational tracheostomy kit (Portex; Hythe, Kent, UK) has largely fallen out of favor. Griggs guidewire dilating forceps technique uses a special forceps (modified Howard Kelly forceps) over a guidewire to result in single step dilatation of soft tissue. The blunt dissection in this procedure was technically more challenging when compared to the Ciaglia technique and was associated with a higher incidence of soft tissue damage and bleeding (30). All PDT kits contain disinfectant solution, tracheostomy introducer, fenestrated drape, lidocaine with epinephrine, scalpel, needles, forceps, needle driver, suture, gauze, a swivel adaptor, and different sized sequential dilators. A tracheostomy tube of choice is separately arranged. In obese patients, an extra-long tracheostomy tube is needed. When using an ultrasound, arrange for a sterile probe cover. If using $\mathrm{FB}$, then having bronchoscopy equipment ready alongside dual suction setup is required as well. Bleeding is known to occur in 2-5\% PDT. Injectable lidocaine with epinephrine, extra sutures and silver nitrate should be part of the setup. It is prudent to have a portable cautery pen or device readily available on standby if worsening bleeding is noted intraoperatively.

\section{Conclusions}

An institutional checklist and dedicated procedural service for critically ill patients helps to reduce errors and harm as well as improve safety. Planning the procedural setup in advance and having all necessary equipment readily available at the patient's bedside ensures timely and successful completion of procedures minimizing overall risk to the patient (Table 2). Interventional pulmonologists are skilled at high-risk ICU procedures and can assist in patient care requiring diagnostic and therapeutic bronchoscopy, thoracentesis, chest tube insertion, difficult airway management, and percutaneous dilatation tracheostomy. 
Table 2 Equipment and preprocedural set up outline for flexible bronchoscopy, pleural procedures and percutaneous tracheostomy in the ICU

Components of preprocedural set up in the intensive care unit

Flexible bronchoscopy

A. Patient

* Supine position

* Bite block

* Swivel adaptor

* Moderate or deep sedation

B. Personnel, procedural team

* Bronchoscopist

* Respiratory Therapist

* Critical Care nurse

C. Equipment

* Airway exam and bronchoalveolar lavage

- Bronchoscope with light source and video display

- Cold saline

- Flexible Yankauer, suction tubing, suction valves and biopsy valves

- Three extra traps for specimen collection

- Local anesthetic (2\% lidocaine jelly/2\% xylocaine $20 \mathrm{~mL}$ vials)

* Bronchoscopy with biopsies (additional equipment)

- Biopsy forceps

- Formalin container

- Epinephrine (to help achieve hemostasis)

Pleural procedures

A. Patient

* Patient position

- Thoracentesis - lateral decubitus in intubated patient

- Chest tube-supine or lateral decubitus position with the head elevated

- TIPC-lateral decubitus position with the head elevated

* Local anesthesia: lidocaine 1\% (×2 vials)

B. Personnel, procedural team

* Proceduralist

* Critical care nurse

C. Equipment

* Ultrasound machine

* ChloraPrep

* Suction set up

* Pleura Vac "Atrium"

* Thoracentesis kit/Chest Tube kit/Pleurx (Denver Kit) Catheter System

Table 2 (continued) 
Table 2 (continued)

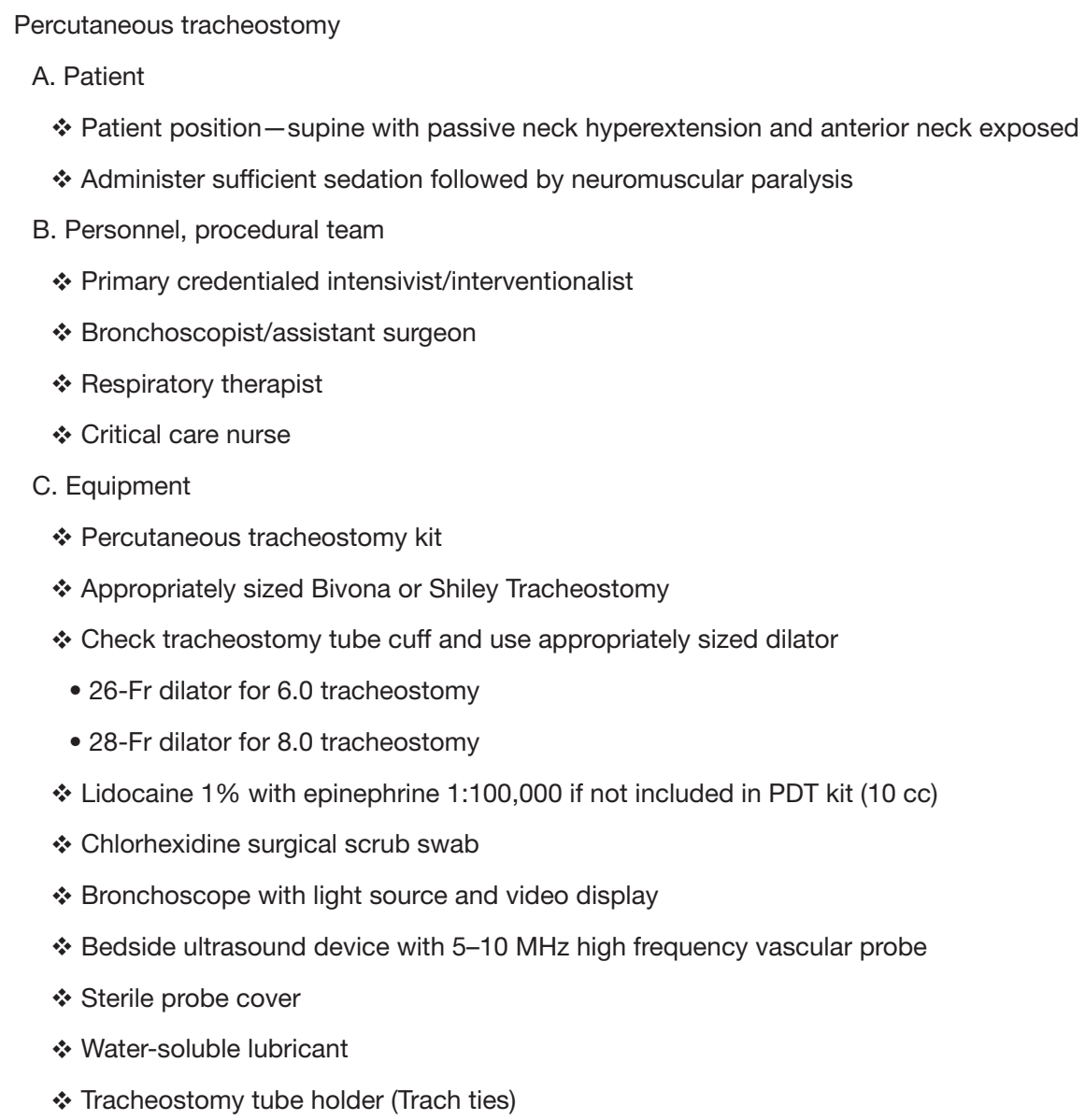

ICU, intensive care unit; PDT, percutaneous dilatational tracheostomy.

\section{Acknowledgments}

Funding: None.

\section{Footnote}

Provenance and Peer Review: This article was commissioned by the editorial office, Fournal of Thoracic Disease for the series "Interventional Pulmonology in the Intensive Care Unit Environment". The article has undergone the external peer review.

Conflicts of Interest: All authors have completed the ICMJE uniform disclosure form (available at http://dx.doi. org/10.21037/jtd-20-3595). The series "Interventional
Pulmonology in the Intensive Care Unit Environment" was commissioned by the editorial office without any funding sponsorship. AS served as the unpaid Guest Editor of the series. All authors have no other conflicts of interest to declare.

Ethical Statement: The authors are accountable for all aspects of the work in ensuring that questions related to the accuracy or integrity of any part of the work are appropriately investigated and resolved.

Open Access Statement: This is an Open Access article distributed in accordance with the Creative Commons Attribution-NonCommercial-NoDerivs 4.0 International 
License (CC BY-NC-ND 4.0), which permits the noncommercial replication and distribution of the article with the strict proviso that no changes or edits are made and the original work is properly cited (including links to both the formal publication through the relevant DOI and the license). See: https://creativecommons.org/licenses/by-nc-nd/4.0/.

\section{References}

1. Nobleza COHS, Pandian V, Jasti R, et al. Outcomes of Tracheostomy With Concomitant and Delayed Percutaneous Endoscopic Gastrostomy in the Neuroscience Critical Care Unit. J Intensive Care Med 2019;34:835-43.

2. Farrow S, Farrow C, Soni N. Size matters: choosing the right tracheal tube. Anaesthesia 2012;67:815-9.

3. Grossman E, Jacobi AM. Minimal optimal endotracheal tube size for fiberoptic bronchoscopy. Anesth Analg 1974;53:475-6.

4. Châteauvieux C, Farah L, Guerot E, et al. Singleuse flexible bronchoscopes compared with reusable bronchoscopes: Positive organizational impact but a costly solution. J Eval Clin Pract 2018;24:528-35.

5. McCahon RA, Whynes DK. Cost comparison of re-usable and single-use fibrescopes in a large English teaching hospital. Anaesthesia 2015;70:699-706.

6. Peroni DG, Boner AL. Atelectasis: mechanisms, diagnosis and management. Paediatr Respir Rev 2000;1:274-8.

7. Abu-Hasan MN, Chesrown SE, Jantz MA. Successful use of bronchoscopic lung insufflation to treat left lung atelectasis. Pediatr Pulmonol 2013;48:306-9.

8. Özdemir C, Sökücü SN, Kocatürk Cİ, et al. Are flexible bronchoscopic cryoextraction practices an alternative to rigid bronchoscopy? Tuberk Toraks 2019;67:15-21.

9. Schmidt LH, Schulze AB, Goerlich D, et al. Blood clot removal by cryoextraction in critically ill patients with pulmonary hemorrhage. J Thorac Dis 2019;11:4319-27.

10. Pitcher HT, Harrison MA, Shaw C, et al. Management considerations of massive hemoptysis while on extracorporeal membrane oxygenation. Perfusion 2016;31:653-8.

11. Fekri MS, Hashemi-Bajgani SM, Shafahi A, et al. Comparing Adrenaline with Tranexamic Acid to Control Acute Endobronchial Bleeding: A Randomized Controlled Trial. Iran J Med Sci 2017;42:129-35.

12. Peralta AR, Chawla M, Lee RP. Novel Bronchoscopic Management of Airway Bleeding With Absorbable Gelatin and Thrombin Slurry. J Bronchology Interv Pulmonol 2018;25:204-11.

13. Sehgal IS, Dhooria S, Ram B, et al. Foreign Body Inhalation in the Adult Population: Experience of 25,998 Bronchoscopies and Systematic Review of the Literature. Respir Care 2015;60:1438-48.

14. Sheski FD, Mathur PN. Endobronchial electrosurgery: argon plasma coagulation and electrocautery. Semin Respir Crit Care Med 2004;25:367-74.

15. Sachdeva A, Pickering EM, Lee HJ. From electrocautery, balloon dilatation, neodymium-doped:yttrium-aluminumgarnet (Nd:YAG) laser to argon plasma coagulation and cryotherapy. J Thorac Dis 2015;7:S363-79.

16. Collins SR, Blank RS. Fiberoptic intubation: an overview and update. Respir Care 2014;59:865-78; discussion 878-80.

17. Hazarika T, O'Donohoe B, Laxton A, et al. Don't forget the bite-block. Anaesth Intensive Care 2010;38:957.

18. Abramson SI, Holmes AA, Hagberg CA. Awake insertion of the Bonfils Retromolar Intubation Fiberscope in five patients with anticipated difficult airways. Anesth Analg 2008;106:1215-7, table of contents.

19. Majid A, Cheng G, Kent M, et al. Evaluation of Rigid Bronchoscopy-Guided Percutaneous Dilational Tracheostomy. A Pilot Study. Ann Am Thorac Soc 2014;11:789-94.

20. Mehta C, Mehta Y. Percutaneous tracheostomy. Ann Card Anaesth 2017;20:S19-25.

21. Gadkaree SK, Schwartz D, Gerold K, et al. Use of Bronchoscopy in Percutaneous Dilational Tracheostomy. JAMA Otolaryngol Head Neck Surg 2016;142:143-9.

22. Raimondi N, Vial MR, Calleja J, et al. Evidence-based guidelines for the use of tracheostomy in critically ill patients. J Crit Care 2017;38:304-18.

23. Gobatto AL, Besen BA, Tierno PF, et al. Comparison between ultrasound- and bronchoscopy-guided percutaneous dilational tracheostomy in critically ill patients: a retrospective cohort study. J Crit Care 2015;30:220.e13-7.

24. Sarıtaş A, Kurnaz MM. Comparison of BronchoscopyGuided and Real-Time Ultrasound-Guided Percutaneous Dilatational Tracheostomy: Safety, Complications, and Effectiveness in Critically Ill Patients. J Intensive Care Med 2017:885066617705641.

25. Even-Tov E, Koifman I, Rozentsvaig V, et al. Preprocedural Ultrasonography for Tracheostomy in Critically Ill Patients: A Prospective Study. Isr Med Assoc J 2017;19:337-40. 
26. Guinot PG, Zogheib E, Petiot S, et al. Ultrasound-guided percutaneous tracheostomy in critically ill obese patients. Crit Care 2012;16:R40.

27. Sangwan YS, Chasse R. A modified technique for percutaneous dilatational tracheostomy: A retrospective review of 60 cases. J Crit Care 2016;31:144-9.

28. Byhahn C, Wilke HJ, Halbig S, et al. Percutaneous tracheostomy: ciaglia blue rhino versus the basic ciaglia technique of percutaneous dilational tracheostomy. Anesth
Analg 2000;91:882-6.

29. Kunz T, Strametz R, Gründling M, et al. Percutaneous tracheostomy in intensive care medicine - Update 2012. Anasthesiol Intensivmed Notfallmed Schmerzther 2012;47:598-604.

30. Cools-Lartigue J, Aboalsaud A, Gill H, et al. Evolution of percutaneous dilatational tracheostomy--a review of current techniques and their pitfalls. World J Surg 2013;37:1633-46.

Cite this article as: Ray AS, Holden VK, Sachdeva A, Nasim F. Equipment and procedural setup for interventional pulmonology procedures in the intensive care unit. $\mathrm{J}$ Thorac Dis 2021;13(8):5331-5342. doi: 10.21037/jtd-20-3595 